Research Article

\title{
Age- and Organ-Specific Differences in Mitochondrial Bioenergetics in Brown Norway Rats
}

\author{
Jignesh D. Pandya, ${ }^{1}$ Matthew Valdez $\mathbb{D}^{2,3}$ Joyce E. Royland, ${ }^{3}$ Robert C. MacPhail, ${ }^{3}$ \\ Patrick G. Sullivan, ${ }^{1}$ and Prasada Rao S. Kodavanti ${ }^{3}{ }^{3}$ \\ ${ }^{1}$ Spinal Cord and Brain Injury Research Center, Department of Neuroscience, University of Kentucky, Lexington, KY 40536, USA \\ ${ }^{2}$ Oak Ridge Institute for Science and Education, U.S. Department of Energy, Oak Ridge, TN 37831, USA \\ ${ }^{3}$ Neurological and Endocrine Toxicology Branch, Public Health and Integrated Toxicology Division, CPHEA/ORD, \\ U.S. Environmental Protection Agency, Research Triangle Park, Durham, NC 27711, USA
}

Correspondence should be addressed to Prasada Rao S. Kodavanti; kodavanti.prasada@epa.gov

Jignesh D. Pandya and Matthew Valdez contributed equally to this work.

Received 16 September 2019; Revised 22 January 2020; Accepted 13 February 2020; Published 1 April 2020

Academic Editor: Jean-Francois Grosset

Copyright (c) 2020 Jignesh D. Pandya et al. This is an open access article distributed under the Creative Commons Attribution License, which permits unrestricted use, distribution, and reproduction in any medium, provided the original work is properly cited.

\begin{abstract}
Mitochondria play a central role in energy homeostasis and act as regulatory checkpoints for downstream metabolic responses and cell senescence processes during an entire life span. Acute or chronic environmental toxicant exposures have shown deleterious organ-specific human health issues at various life stages. Since mitochondria are a prime target for ensuing cellular bioenergetics responses and senescence, it is essential to understand mitochondrial bioenergetic responses in different organs over multiple life stages. Therefore, in the present study, we evaluated mitochondrial bioenergetic parameters in the liver, lung, and heart in four diverse age groups (young: 1 month; adult: 4 months; middle-aged: 12 months; old-aged: 24 month) using male Brown Norway rats as a model of aging ( $n=5$ sample size/organ/age group) and compared them with our previously published results on brain. Real-time mitochondrial bioenergetic parameters (i.e., State III, State IV, and State V) were measured using the Seahorse Extracellular Flux Analyzer. Additionally, mitochondrial enzyme pyruvate dehydrogenase complex (PDHC), Complex I, Complex II, and Complex IV activities were measured using Synergy HT plate reader. Our results indicated that nearly in all parameters, significant age- and organ-specific interactions were observed. We observed age-specific declines in State III (i.e., ATP synthesis rate) responses in both the heart and lung, where opposite was observed in the liver as age advances. Across the age, the heart has highest enzyme activities than the liver and lung. Interestingly, heart and liver mitochondrial bioenergetic rates and enzyme activities remain higher than the lung, which specifies their higher metabolic capabilities than the lung. Amongst all, bioenergetic rates and enzyme activities in the lung remain lowest suggesting the lung may display higher vulnerability and lower resilience to environmental toxicants during aging than other organs tested here. Overall, these age- and organ-specific findings may facilitate a more contextualized understanding of mitochondrial bioenergetic outcomes when considering the interactions of age-related sensitivities with exposure to chemical stressors from the environment.
\end{abstract}

\section{Introduction}

Mitochondria are known as the "powerhouse" of the cell. Metabolic breakdown of complex foods yields glucose, fatty acid, or amino acids via digestion, and then these components are further oxidatively metabolized by mitochondria in cells based on energy needs of an organ. Since glucose is the primary energy source of all organs, it is further metabolized to pyruvate in the cytosol via glycolysis. The pyruvate dehydrogenase complex (PDHC) enzyme is an entry point of the Krebs cycle where it feeds acetyl groups derived from pyruvate to coenzyme A, thereby initiating cyclic aerobic oxidation of substrates and generating reducing equivalents, i.e., $\mathrm{NADH}$ and $\mathrm{FADH}_{2}$. Both of them donate 
electrons to electron transport chain (ETC) Complex enzymes I and II, respectively. Electrons are then transported through Complexes III and IV to the Complex IV as final acceptor of $\mathrm{O}_{2}$ to generate water. During this aerobic respiration process, the flow of electrons is coupled to the transfer of protons from the mitochondrial matrix to the intermembrane space and establish the mitochondrial membrane potential $\left(\Delta \Psi_{\mathrm{m}}\right)$, which is used by Complex $\mathrm{V}$ (i.e., ATP synthase) to generate energy in the form of adenosine triphosphate (ATP).

Although highly orchestrated and conserved, this ETC electron transfer process usually generates minimal electron leaks ( $\sim 1 \%$ of respiration) in healthy condition. This electron leakage targets oxygen and nitrogen molecules in the vicinity and may yield toxic products such as reactive oxygen/nitrogen species (ROS/RNS) [1, 2]. The cellular antioxidant system regulates these ROS/RNS levels. In recent years, ROS have been described in signaling pathways that are potentially beneficial to cells [3]; however, their overproduction is more commonly known to damage proteins, lipids, and DNA. Due to these complex and central cellular processes governed by mitochondria, several theories of aging have been discussed earlier. They are outlined based on rate-ofliving theory: high metabolic rate= decreased life span [4]; mitochondrial decline theory: decreased mitochondrial function=decreased life span [5]; uncoupling-to-survive theory: increased uncoupling activity = increased life span [6]; mitohormesis: moderate, chronic ROS production $=$ increased life span [7]; free radical theory: increased ROS = decreased life span [8]; and damage theory: accumulated damage from imperfect biomolecules $=$ decreased lifespan [9] .

The rate of reported acute and long-term exposures of toxic drugs and pollutants on mitochondria function has increased over recent decades $[10,11]$. In fact, mitochondrial dysfunctions are some of the most predictable findings across high priority chemicals screening assays performed at the National Toxicology Program [12]. While mitotoxicants such as pentachlorophenol [13], carbon monoxide [14], rotenone [15], and oligomycin [16] have been known for decades, newer chemicals such as pesticides, herbicides, cigarette and cookstove smoke, and even air pollution have all been found to impact mitochondria function [17]. For example, exposure to trichloroethylene, an environmental toxicant used in producing refrigerants and to remove grease from metal products, produces mitochondrial complex I dysfunction which is observed in a neurological disorder, i.e., both Parkinsonism patients and a rat model [18, 19]. In addition to toxicant interactions, mitochondria are also central to other pathologies. Mitochondrial dysfunction has been implicated in the etiology of heart failure [20]. Specific patterns of irregular mitochondrial signatures are indicators of several lung disease such as asthma, chronic obstructive pulmonary disease, and even cancer [21]. Within the liver, the cause of nonalcoholic fatty liver disease is unknown, but the prevailing theories suggest that decreased capacity of mitochondria to oxidize fatty acids may be involved in the onset of the disease [22].

Given the role of mitochondria in both aging and toxicity, it is essential to characterize mitochondrial bioenergetics across all life stages in order to fully understand toxicological adverse outcome pathways. In addition, effects of mitochondrial dysfunction have empirically been found to be cell- and tissue-specific [10] which complicate the matter further. In this report, we present an in-depth, comprehensive study to advance our understanding of the role of mitochondrial bioenergetic function in aging. The study was carried out in the Brown Norway rat, a model of healthy aging. Mitochondrial bioenergetics and enzyme activities were evaluated at multiple ages from young, adult, middle-aged, and old animals (1, 4, 12, and 24 months, respectively) in different organs (heart, liver, and lung) and compared with our previously published data in the brain using frontal cortex as a representative [23]. Mitochondrial respiration parameters State III rate (ATP synthesis capacity), State IV rate (proton leakage), and State $\mathrm{V}$ rate (maximal electron transport) were measured together with mitochondrial enzyme (PDHC and Complexes I, II, and IV) activities. Taken together, these parameters reveal the overall organ-specific mitochondrial bioenergetic efficiency across lifespan. The comprehensive design of this study will provide beneficial characterization of age-related metabolic status changes in individual organs for future studies to assess the adverse effects of mitotoxicants (environmental chemicals) at different life stages. Additionally, knowledge gained in this study will aid in the understanding of age-induced sensitivity towards environmental chemicals.

\section{Materials and Methods}

2.1. Animals. Male Brown Norway rats from four different life stages were examined (i.e., 1 month (1M): young, 4 months (4M): adult, 12 months (12M): middle-aged, and 24 months (24M): old-aged) in this study. Animals $(N=5-6$ rats/group) were purchased from the National Institutes of Health (NIA) animal facilities at their appropriate age, less than 2 weeks prior to the actual experiment. All animal protocol and procedures were approved by the University of Kentucky Institutional Animal Care and Use Committee. Animals were maintained at University of Kentucky animal facilities on a regular 12-hour light/12-hour dark cycle in temperature-controlled rooms $\left(25^{\circ} \mathrm{C}\right)$ for the first week to acclimate to the environment. Animals were pair-housed and provided with ad lib rat chow and water. To limit age variability within groups, all animals were used within 3 days for the $1 \mathrm{M}$ age group and to a maximum of 14 days for the $4 \mathrm{M}, 12 \mathrm{M}$, and $24 \mathrm{M}$ age groups. After the 1-week acclimatization, all animals were randomly assigned dates of sacrifice. Two animals per day (except for $1 \mathrm{M}$ animals where 2 animals were pooled per single sample giving 4 animals/day) were processed for organ-specific mitochondrial isolation and bioenergetic parameter measurements.

2.2. Isolation of Mitochondria. Mitochondria from 3 visceral organs (i.e., heart, liver, and lung) were isolated together under identical experimental conditions on each experimental day using an established and previously published ficoll-based mitochondrial ultrapurification procedure 
[24-27]. Mitochondria from five brain regions were also isolated at the same time from the same cohort of animals and the results were published earlier [23]. Throughout the mitochondrial isolation period, samples were kept at $4^{\circ} \mathrm{C}$ on ice, unless otherwise specified. Animals were asphyxiated with $\mathrm{CO}_{2}$ and rapidly decapitated. Following decapitation, the heart, liver, and lung were rapidly removed and placed in a beaker of ice-cold $\left(4^{\circ} \mathrm{C}\right)$ mitochondrial isolation buffer (MIB) composed of $215 \mathrm{mM}$ Mannitol, $75 \mathrm{mM}$ sucrose, $0.1 \%$ BSA, $1 \mathrm{mM}$ EGTA, and $20 \mathrm{mM}$ HEPES at $\mathrm{pH}$ 7.2. All organs of interest were rapidly dissected apart, placed in prelabeled $5 \mathrm{ml}$ tubes containing $1 \mathrm{ml}$ of MIB buffer, and homogenized. Tissue homogenates were centrifuged at $1300 \times \mathrm{g}$ for $3 \mathrm{~min}$ to remove cell debris and nuclei, and the collected supernatant was then centrifuged at $13,000 \times \mathrm{g}$ for $10 \mathrm{~min}$ to pellet the mitochondria. The resultant crude mitochondrial pellet was then resuspended in $500 \mu \mathrm{l}$ of MIB and placed on top of discontinuous ficoll gradient (layered $2 \mathrm{ml}$ of $7.5 \%$ ficoll solution on top of $2 \mathrm{ml}$ of $10 \%$ ficoll solution) and centrifuged at $100,000 \times \mathrm{g}$ for 30 min using a Beckman ultracentrifuge with SW55Ti rotor (Beckman Coulter, IN, USA). Ultrapure mitochondrial pellets formed at the bottom were carefully removed and were resuspended in $2 \mathrm{ml}$ MIB (without $1 \mathrm{mM}$ EGTA buffer) and centrifuged at $10,000 \times \mathrm{g}$ for $10 \mathrm{~min}$. The washed mitochondrial pellets were finally resuspended in $\mathrm{MIB}^{-}$to a final concentration of $\sim 10 \mu \mathrm{g} / \mu \mathrm{l}$ mitochondrial protein (volume varied depending on organs). Mitochondrial protein concentration was determined using the BCA protein assay kit measuring absorbance at $562 \mathrm{~nm}$ with BioTek Synergy HT plate reader (Winooski, VT, USA).

2.3. Mitochondrial Bioenergetics Measurements. Measurements of mitochondrial bioenergetics in isolated mitochondrial samples were performed immediately after isolation using a slightly modified published protocol of Seahorse XF24 Extracellular Flux Analyzer (Agilent Inc., Santa Clara, CA) [28-31].In brief, stock solutions of mitochondrial substrates of $500 \mathrm{mM}$ pyruvate, $250 \mathrm{mM}$ malate, and $30 \mathrm{mM}$ ADP plus assay solutions of $1 \mathrm{mg} / \mathrm{ml}$ oligomycin- $\mathrm{A}, 1 \mathrm{mM}$ carbonyl cyanide-4 (trifluoromethoxy) phenylhydrazone (FCCP), $1 \mathrm{mM}$ rotenone, and $1 \mathrm{M}$ succinate were prepared. The $\mathrm{pH}$ was adjusted to 7.2 , and the solutions were stored at $-20^{\circ} \mathrm{C}$. The day before the planned experiment, a 24 well dualanalyte sensor cartridge was hydrated and kept in a non- $\mathrm{CO}_{2}$ incubator at $37^{\circ} \mathrm{C}$. On the scheduled day of the experiment, the sensor cartridge ports A to D were loaded with the appropriate mitochondrial substrates or inhibitors (freshly prepared 10X working concentration made from stocks) and sequentially injected into the assay plate according to the protocol procedure to reach a final concentration of the compound (1X) in each well. All mitochondrial working stocks were prepared in respiration buffer (RB) composed of $215 \mathrm{mM}$ mannitol, $75 \mathrm{mM}$ sucrose, $0.1 \% \mathrm{BSA}, 20 \mathrm{mM}$ HEPES, $2 \mathrm{mM} \mathrm{MgCl}_{2}$, and $2.5 \mathrm{mM} \mathrm{KH}_{2} \mathrm{PO}_{4}$ at $\mathrm{pH} 7.2$ and stored at $4^{\circ} \mathrm{C}$. The amount of substrates/inhibitors loaded for each port is based upon the initial $500 \mu \mathrm{l} \mathrm{RB}$ volume in the mitochondrial plate as follows: Port A: $50 \mu \mathrm{l}$ (mixture of pyruvate, malate, and ADP), Port B: $55 \mu \mathrm{l}$ (oligomycin A), Port C: $60 \mu \mathrm{l}$ (FCCP), and Port D: $65 \mu \mathrm{l}$ (rotenone and succinate). Once the sensor cartridge was loaded with all of the experimental reagents, it was placed into the Seahorse XF24 Flux Analyzer for automated calibration.

Seahorse Standard XF24 assay plates were utilized for mitochondrial analysis. Mitochondria $(15 \mu \mathrm{g})$ from three visceral organs were analyzed together on a single plate. Purified mitochondria as described above were added to RB buffer to give a final concentration of $15 \mu \mathrm{g}$ mitochondrial protein in $50 \mu \mathrm{l} \mathrm{RB}$ and added to experimental wells. Background control wells contained $50 \mu \mathrm{l}$ of RB without mitochondria. Loaded XF24 plates were centrifuged at $3,000 \mathrm{rpm}$ for 4 minutes at room temperature in a tabletop centrifuge to attach mitochondria to the well bottom. Following centrifugation, $450 \mu \mathrm{l}\left(37^{\circ} \mathrm{C}\right)$ of prewarmed $\mathrm{RB}$ was gently added to each well for a final volume of $500 \mu \mathrm{l}$ per well. Following sensor cartridge calibration, plates were placed into the Seahorse XF24 Extracellular Flux analyzer for mitochondrial bioenergetics analysis.

An optimized protocol was utilized for the analysis of bioenergetics function in purified mitochondria using the Seahorse Biosciences XF24 Flux Analyzer. The protocol contains cyclic steps of a cartridge probe calibration, baseline readings, sequential injections of substrates/inhibitors via ports $\mathrm{A}$ thru $\mathrm{D}$, substrates mixing in the assay system, a delay for well $\mathrm{O}_{2}$ equilibration, and then measurement of the oxygen consumption rates (OCR) as elaborated upon previously [28, 30, 31]. The OCR rates of State III responses in presence of $5 \mathrm{mM}$ pyruvate, $2.5 \mathrm{mM}$ malate, and $1 \mathrm{mM}$ ADP (Port A) were measured followed by State IV response in presence of $1 \mu \mathrm{M}$ oligomycin A (Port B). Sequentially, State $\mathrm{V}_{\mathrm{FCCP}}$ and State $\mathrm{V}_{\text {Succ }}$ OCR rates were recorded automatically in presence of $4 \mu \mathrm{M}$ carbonyl cyanide 4 -(trifluoromethoxy)phenyl hydrazone (i.e., FCCP) (Port C) and $0.1 \mu \mathrm{M}$ rotenone plus $10 \mathrm{mM}$ of succinate (Port D), respectively. After experiment, the Seahorse file was configured to display OCR rates for Ports A-D in a point-to-point mode. The highest OCR rates for ports $\mathrm{A}-\mathrm{D}$ were used to perform data analysis. Data are reported as OCR for the various brain regions isolated from the four age groups.

\subsection{Mitochondrial Enzyme Activity Assessments.} Mitochondrial Krebs cycle gatekeeper and electron transport chain (ETC) enzyme activities were performed with an automated 96-well microplate reader (Bio-Tek Instruments Inc., Winooski, VT) as described in previously published articles [32-34]. For all enzyme assays, stored mitochondrial samples were diluted to $1 \mu \mathrm{g} / \mu \mathrm{l}$ in $10 \mathrm{mM} \mathrm{KH}_{2} \mathrm{PO}_{4}$ followed by freeze-thaw and sonication together three times to release enzymes before measuring enzyme activities for all samples on single plate at $37^{\circ} \mathrm{C}$.

The mitochondrial Krebs cycle gatekeeper enzyme pyruvate dehydrogenase complex (PDHC) enzyme activity was measured by the production of $\mathrm{NADH}$ as previously described [32,33], with slight modifications. Ficoll-purified 
mitochondrial proteins $(20 \mu \mathrm{g} /$ well $)$ were added in triplicate to wells containing $100 \mu \mathrm{l}$ total assay volume of reaction buffer $(50 \mathrm{mM} \mathrm{KCl}, 10 \mathrm{mM}$ HEPES $\mathrm{pH}$ 7.4, $0.3 \mathrm{mM}$ thiamine pyrophosphate, $10 \mu \mathrm{M} \mathrm{CaCl}_{2}, 0.2 \mathrm{mM} \mathrm{MgCl}, 5 \mathrm{mM}$ pyruvate, $1 \mu \mathrm{M}$ rotenone, and $0.2 \mathrm{mM} \mathrm{NAD}^{+}$) in a $96-$ well format. The enzyme reaction was started by addition of $0.14 \mathrm{mM}$ Coenzyme A/well. Measurements were taken at $1 \mathrm{~min}$ intervals for $3 \mathrm{~min}$ at Excitation/Emission (Ex $\lambda$ $340 \mathrm{~nm} / \mathrm{Em} \lambda 460 \mathrm{~nm}$ ). The PDHC activity was calculated and expressed as $\mathrm{nmol} / \mathrm{min} / \mathrm{mg}$ mitochondrial protein.

The Complex I (NADH dehydrogenase) enzyme activity was performed in triplicate in a reaction buffer of $25 \mathrm{mM}$ $\mathrm{KH}_{2} \mathrm{PO}_{4}$ (pH 7.2) containing $5 \mathrm{mM} \mathrm{MgCl}, 1 \mathrm{mM} \mathrm{KCN}$, $1 \mathrm{mg} / \mathrm{ml} \mathrm{BSA}$, and $150 \mu \mathrm{M}$ NADH. Mitochondrial proteins $(20 \mu \mathrm{g})$ were added to the reaction buffer in $100 \mu \mathrm{l}$ of assay volume, and the assay was performed in the presence or absence of rotenone $(10 \mu \mathrm{M})$ to determine the rotenoneinsensitive and the rotenone-sensitive Complex I enzyme activity. The rotenone-insensitive rate of $\mathrm{NADH}$ oxidation was subtracted to obtain rotenone-sensitive activity. The reaction was started by the addition of $50 \mu \mathrm{M}$ of coenzyme $\mathrm{Q} 1$. The disappearance of $\mathrm{NADH}$ over time was measured at $1 \mathrm{~min}$ intervals for $20 \mathrm{~min}$. The assay was performed at $\operatorname{Ex} \lambda$ $340 \mathrm{~nm} / \mathrm{Em} \lambda 460 \mathrm{~nm}$. Enzyme activity was calculated and expressed as $\mathrm{nmol} / \mathrm{min} / \mathrm{mg}$ mitochondrial protein.

Mitochondrial Complex II (succinate dehydrogenase) enzyme activity was determined by measuring the change in absorbance of a dye, 2,6-Dichlorophenol Indophenol (DCIP), at $600 \mathrm{~nm}$ using a BioTek Synergy HT plate reader (BioTek, Winooski, VT). In brief, $20 \mu \mathrm{g}$ of mitochondrial protein was added to $200 \mathrm{mM} \mathrm{KH}_{2} \mathrm{PO}_{4}$ pH 7.0 assay buffer containing $20 \mathrm{mMK}$-Succinate, $10 \mu \mathrm{M}$ EDTA, $0.01 \% \mathrm{Tx}-$ 100 , and $1 \mu \mathrm{g}$ coenzyme Q10 in a total assay volume of $100 \mu \mathrm{l} /$ well in triplicate. An approximately $20 \mathrm{mg} \%$ DCIP dye was added to obtain initial absorbance readings between $0.8-1.0$ at $600 \mathrm{~nm}$. The decrease in absorbance was measured constantly for $15 \mathrm{~min}$ and the activity was expressed as in terms of nmols of succinate oxidized $/ \mathrm{min} / \mathrm{mg}$ mitochondrial protein $[25,35]$.

The mitochondrial Complex IV (cytochrome c oxidase) activity was measured in $10 \mathrm{mM} \mathrm{KH}_{2} \mathrm{PO}_{4}$ buffer ( $\mathrm{pH} 7.4$ ) coincubated with $20 \mu \mathrm{g}$ mitochondrial protein in triplicate. The reaction was initiated by adding $50 \mu \mathrm{M}$ reduced cytochrome c (final total assay volume of $100 \mu \mathrm{l}$ ). The rate of oxidation of cytochrome $c$ was measured as the decreased absorbance of reduced cytochrome $\mathrm{c}$ at $550 \mathrm{~nm}$ for $2 \mathrm{~min}$. The rate constant $(\mathrm{K})$ of oxidation of cytochrome $\mathrm{c}$ was calculated and expressed as $\mathrm{K} / \mathrm{min} / \mathrm{mg}$ mitochondrial protein $[28,36]$.

2.5. Statistical Analysis. The values represented in Figures $1-4$ are mean \pm SEM $(n=5-6$ sample size/organ/age group). Statistical analysis was performed using RStudio [37]. Raw data were imported and organized and column statistics were generated with the dplyr package [38]. Linear models were constructed using the $\operatorname{lm}()$ function in the $\mathrm{R}$ base package. Models were defined as the main effects and interaction of age and organ for each parameter. Normality and homogeneity of variances assumptions were tested with Shapiro-Wilk test and Levene's test, respectively. When subsets of data did not satisfy normality and homogeneity of variance assumptions, the linear models were modified by log-transforming the data. All subsets of data, except Complex I and II, were log-transformed.

All linear models were analyzed via two-way analysis of variance. Significant main effects and interactions were followed with a post hoc multiple comparisons test. Pairwise comparisons of age and organ groups were performed using Fisher's least significant difference post hoc test. Statistical significance was defined at $p<0.05$ for all determinations. All figures were generated using the ggplot2 [39] and cowplot [40] packages.

\section{Results}

In the present study, we used the Seahorse Extracellular Flux Analyzer (Seahorse Bioscience XF-24) to analyze bioenergetics in 3 visceral organs and compared them with frontal cortex (representative of brain) in four different life stages (young: 1M; adult: 4M; middle-aged: $12 \mathrm{M}$; and old aged: 24M) of Brown Norway rats. A typical pattern of oxygen consumption rate (OCR, a measure of oxidative phosphorylation) of brain mitochondria illustrates age-specific changes in mitochondrial respiration rates as shown in Figure 5. All other figures (Figure 1-4) depict average activities from 5-6 biological replicates by organ across age. Mitochondrial bioenergetic parameters included were State III respiration rate (ATP synthesis capacity), State IV respiration rate (proton leaking), and State $\mathrm{V}$ respiration rates (maximal electron transport) in presence of Complex I or Complex II substrates (Figures 1 and 2). Additionally, we measured mitochondrial PDHC, Complex I, Complex II, and Complex IV enzyme activities to observe comprehensive picture of differences in bioenergetic profile following different life stages preclinically (Figures 3 and 4).

3.1. Age- and Organ-specific Differences in Mitochondrial Bioenergetics. Age- and organ-specific differences in mitochondrial bioenergetics were present in nearly all organs. Two-way ANOVA analysis of State III respiration rate (Figure 1(a)), which is related to ATP synthesis rate, indicated a significant interaction of age and organ $\left(F_{9,68}=5.822\right.$, $\left.p=5.612 e^{-6}\right)$ throughout life stages, with all organs displaying higher average respiration rates compared to lung as determined by post hoc pairwise comparisons. These differences appear to arise after 1 month in the lung, with all State III respirations rates dropped near to 200 OCR as the animal aged. There was also a trend of decreasing State III respiration in the heart, with the largest change observed between $1 \mathrm{M}$ and $12 \mathrm{M}$. Interestingly, this decreased state III respiration rate in the heart rebounded after $24 \mathrm{M}$. State III respiration remains consistent between $1 \mathrm{M}$ to $4 \mathrm{M}$ in the liver with a significant increase noted at $12 \mathrm{M}$ and $24 \mathrm{M}$.

State IV respiration rate (Figure $1(\mathrm{~b})$ ), which represents proton leakage through the mitochondrial membrane, also had a significant interaction of age and organ as indicated by 


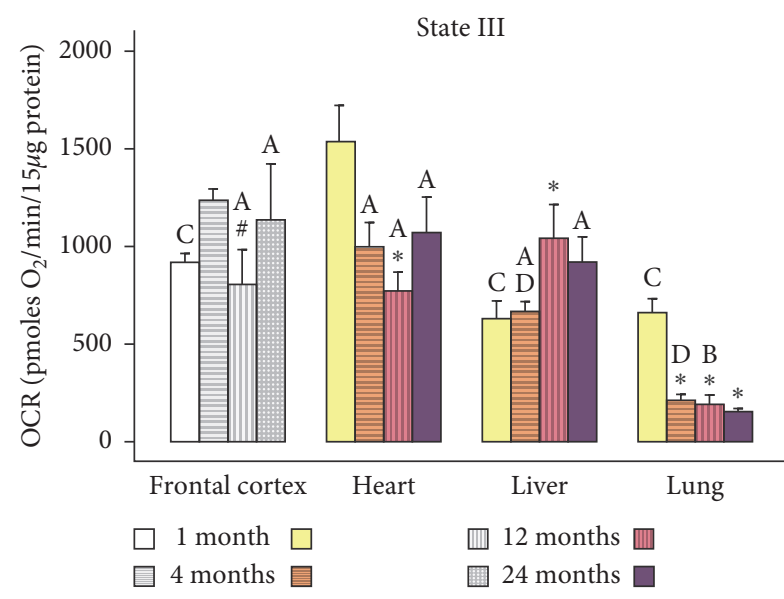

(a)

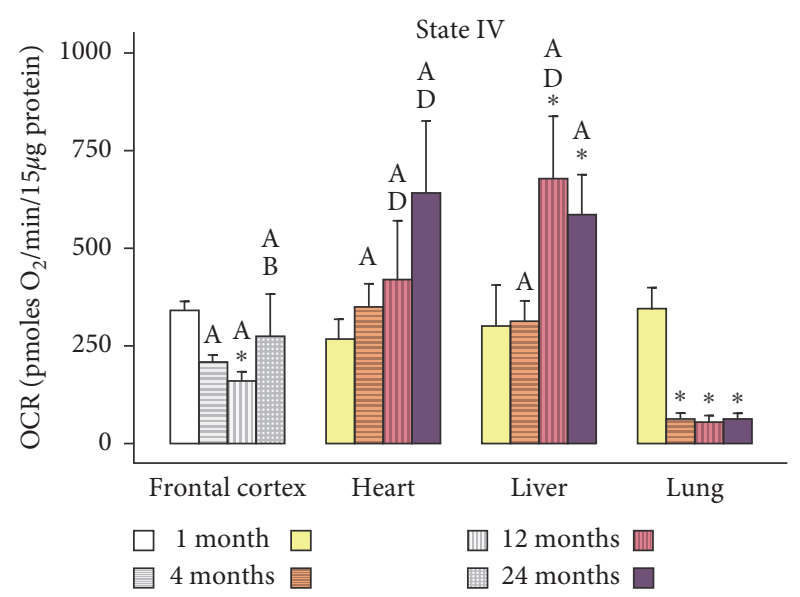

(b)

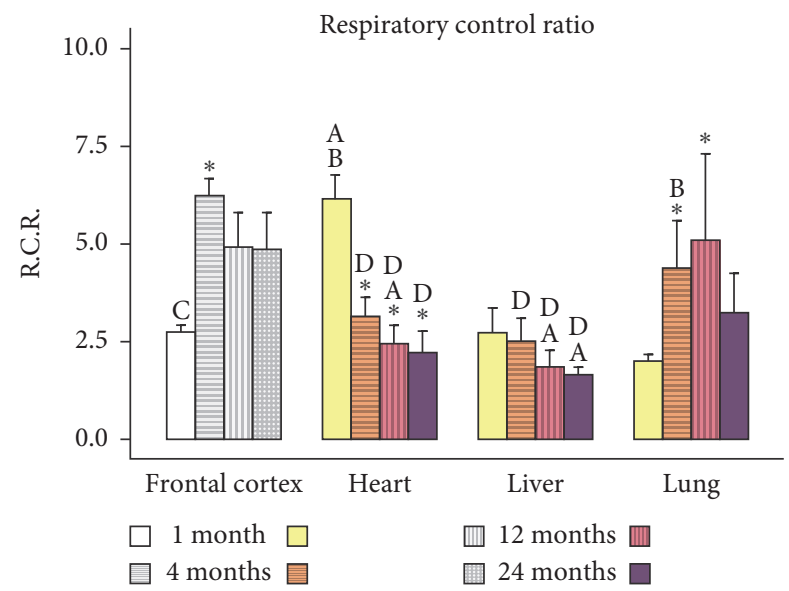

(c)

Figure 1: Age-dependent changes in State III (a) and State IV respiration (b) rates and respiratory control ratio (c) in different organs. Values are expressed as means \pm SEM from $n=5-6$ observations/group. The differences between pairwise comparisons were considered statistically significant at $p<0.05$. * Significantly different from $1 \mathrm{M}$ rats; ${ }^{*}$ significantly different from $4 \mathrm{M}$ rats; ${ }^{\mathrm{A}}$ significantly different from lung; ${ }^{B}$ significantly different from liver; ${ }^{C}$ significantly different from heart; ${ }^{D}$ significantly different from frontal cortex; SEM, standard error of the mean. Grey-scale textured bars represent frontal cortex data from our previous report (adapted from Pandya et al. [23], with permission).

two-way ANOVA $\left(F_{9,68}=4.987, p=3.743 e^{-5}\right)$. State IV respiration in the heart steadily increased over time, but this trend was not significant may be due to higher standard errors in experiments within group due to limited sample size used in the study. Matching the pattern of State III respiration in the liver, State IV also displayed a significant increase at $12 \mathrm{M}$ that persisted until $24 \mathrm{M}$. Just as in State III respiration, the lung had an overall lower average State IV respiratory rates compared to all other organs. Also consistent with the State III respiration in the lung, proton leakage decreased after $1 \mathrm{M}$ similarly across all ages. Again, when compared organ-specific effects of State IV across age, we observed similar pattern of State IV as we have seen in state III respiration rates. More specifically, lung mitochondrial State IV rates were comparable with heart and liver in $1 \mathrm{M}$. It is interesting to note that, as the heart and liver are the major metabolic organs of the body, their mitochondria showed higher State III and State IV respiration rates compared tothe lung, which has the least respiratory capacity at $1 \mathrm{M}$ age. As age increases, the $4 \mathrm{M}-24 \mathrm{M}$ group of lung mitochondria showed significantly less proton leakage than the heart and liver.

We measured mitochondrial State $\mathrm{V}$ respiration rate (maximal electron transport) by applying an uncoupling agent (FCCP) through Port $\mathrm{C}$ that triggers collapse of the proton gradients across mitochondrial inner membrane and disrupts the mitochondrial membrane potential in combination with either a Complex I or Complex II specific substrate addition (Figure 2). The State $\mathrm{V}_{1}$ (Figure 2(a)) measures the uncoupling rates in the presence of the Complex 1 substrates pyruvate and malate. The State $V_{2}$ (Figure 2(b)) measures the uncoupling rates in the presence of the Complex II substrate succinate and the Complex I inhibitor, and rotenone was added through port D to block complex I driven respiration. These OCR rates measure maximal respiratory capacity of ETC through Complex I or Complex II, since ETC respiration is delinked with oxidative phosphorylation using ATP synthase inhibitor oligomycin in the Seahorse assay chamber. Like the other parameters, 


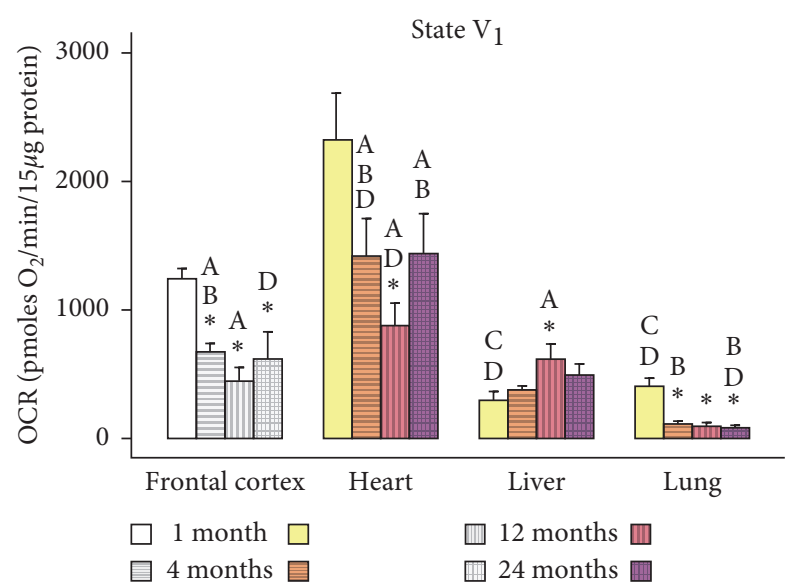

(a)

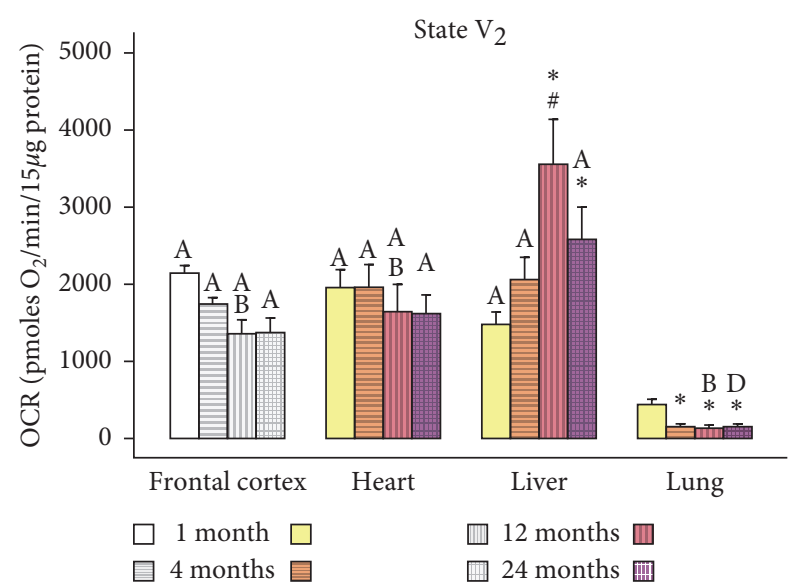

(b)

FIgURE 2: Age-dependent changes in State $V_{1}(a)$ and State $V_{2}$ OCR rates (b) in different organs. Values are expressed as means \pm SEM from $n=5-6$ observations/group. The differences between pairwise comparisons were considered statistically significant at $p<0.05 .{ }^{*}$ Significantly different from $1 \mathrm{M}$ rats; ${ }^{\mathrm{A}}$ significantly different from lung; ${ }^{\mathrm{B}}$ significantly different from liver; ${ }^{\mathrm{C}}$ significantly different from heart; ${ }^{\mathrm{D}}$ significantly different from frontal cortex; SEM, standard error of the mean. Grey-scale bars represent frontal cortex data from our previous report (adapted from Pandya et al. [23], with permission).

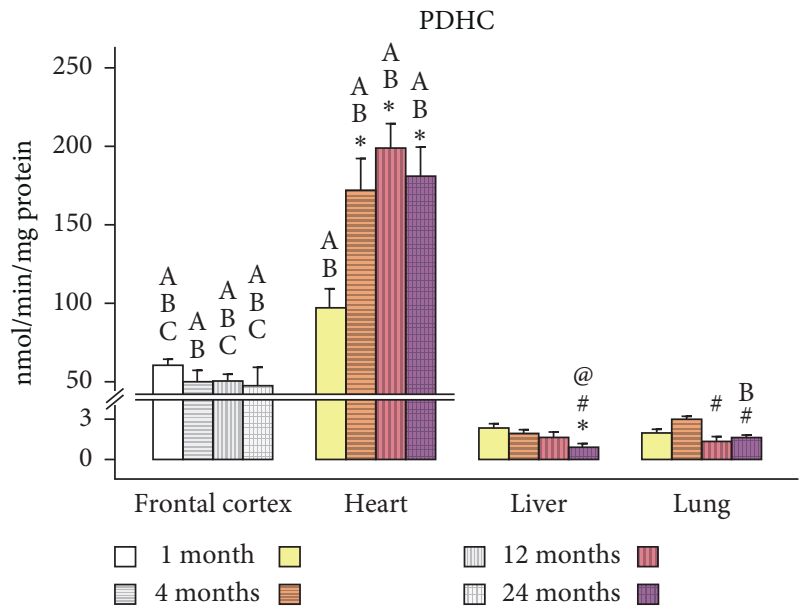

(a)

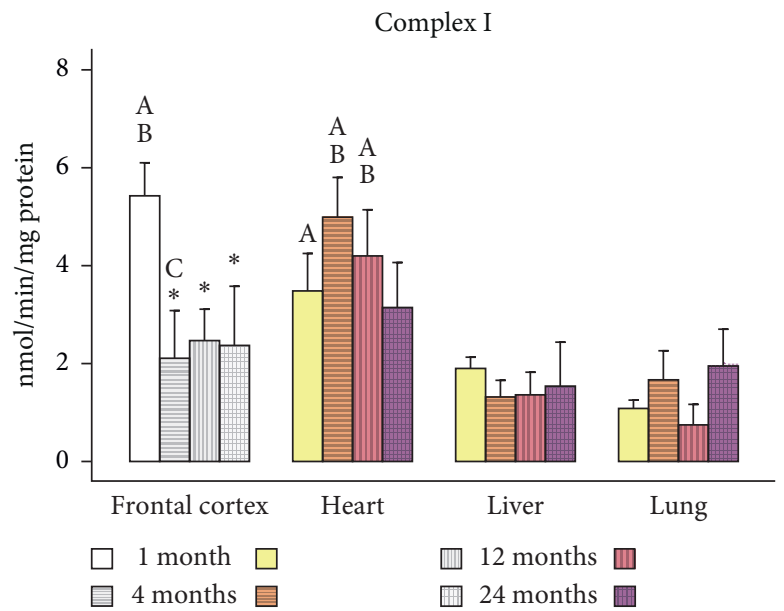

(b)

Figure 3: Age-dependent changes in activities of PDHC (a) and Complex I (b) enzymes in different organs. Values are expressed as means \pm SEM from $n=5$ observations/group. The differences between pairwise comparisons were considered statistically significant at $p<0.05 .{ }^{*}$ Significantly different from $1 \mathrm{M}$ rats; ${ }^{\#}$ significantly different from $4 \mathrm{M}$ rats; ${ }^{\circledR}$ significantly different from $12 \mathrm{M}$ rats; ${ }^{\mathrm{A}}$ significantly different from lung; ${ }^{\mathrm{B}}$ significantly different from liver; ${ }^{\mathrm{C}}$ significantly different from heart; SEM, standard error of the mean. Grey-scale bars represent frontal cortex data from our previous report (adapted from Pandya et al. [23], with permission). Note the $y$-axis scale difference between frontal cortex and heart versus liver and lung.

two-way ANOVA indicated a significant interaction of age and organ for State $\mathrm{V}_{1}$ respiration $\left(F_{3,68}=4.531 ; p=0.0001\right)$ (Figure 2(a)). There was a trend of decreasing State $\mathrm{V}_{1}$ respiration in the heart, with a significant difference between $1 \mathrm{M}$ and $12 \mathrm{M}$, whereas at $24 \mathrm{M}$, the rate increased. Conversely, State $V_{1}$ respiration in the liver increased with age with a significant difference between $1 \mathrm{M}$ and $12 \mathrm{M}$. Then again, in the lung after $1 \mathrm{M}$, there was a significant decrease in State $V_{1}$ respiration that persisted until 24M. Lung State $V_{1}$ respiration followed similar pattern as state III respiration.
The maximal respiratory capacity was significantly declined at later age between $4 \mathrm{M}-24 \mathrm{M}$ as compared to $1 \mathrm{M}$ age group. When compared organ specificity, in young $1 \mathrm{M}$ group, the heart State $V_{1}$ rates were higher than both liver and lung. Subsequently, between $4 \mathrm{M}-24 \mathrm{M}$ period, the heart State $\mathrm{V}_{1}$ rates remain highest than moderately respired liver, followed by the least respired lung.

Like State $\mathrm{V}_{1}$, two-way ANOVA revealed a significant interaction of age and organ $\left(F_{9,68}=5.104, p=2.855 e^{-5}\right)$ in State $\mathrm{V}_{2}$ (Figure 2(b)), Complex II driven respiration. In the 


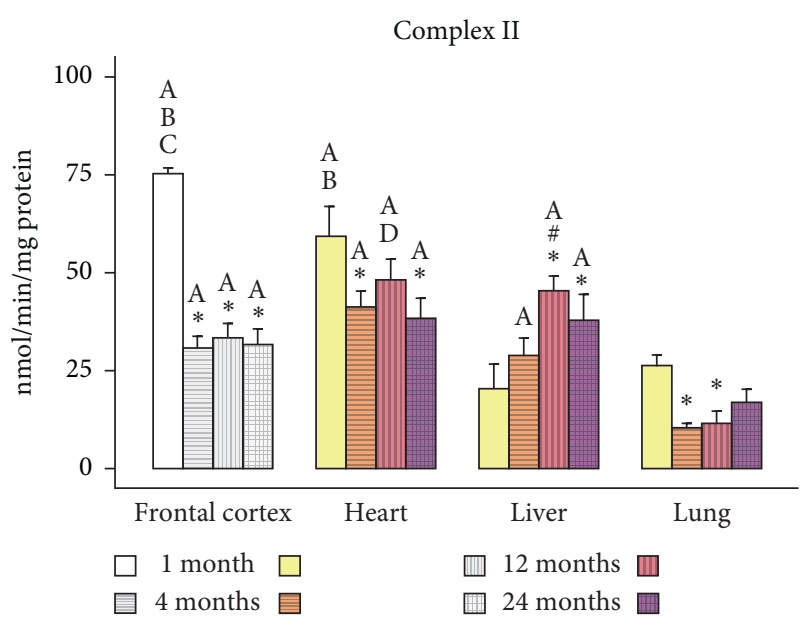

(a)

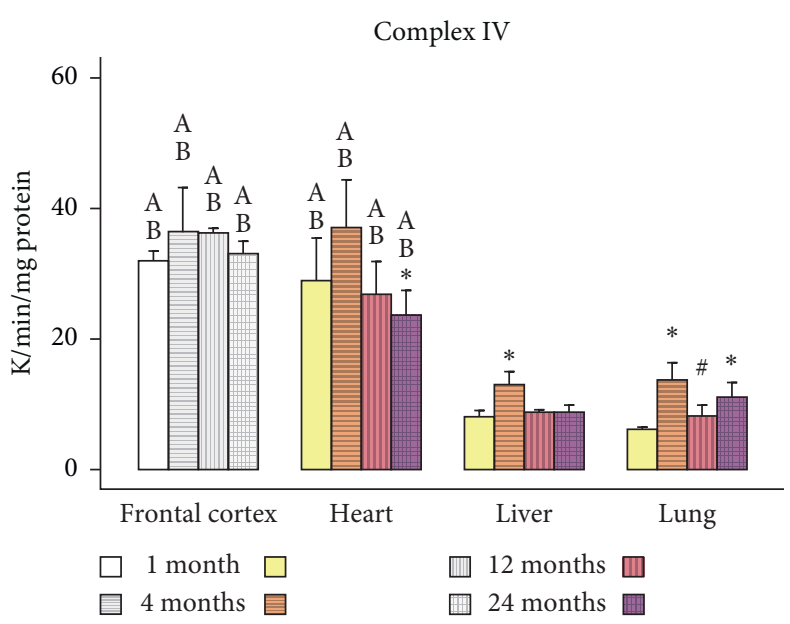

(b)

FIGURE 4: Age-dependent changes in activities of Complex II (a) and Complex IV (b) enzymes in different organs. Values are expressed as means \pm SEM from $n=5$ observations/group. The differences between pairwise comparisons were considered statistically significant at $p<0.05$. *Significantly different from $1 \mathrm{M}$ rats; ${ }^{\#}$ significantly different from $4 \mathrm{M}$ rats; ${ }^{\mathrm{A}}$ significantly different from lung; ${ }^{\mathrm{B}}$ significantly different from liver; ${ }^{\mathrm{C}}$ significantly different from heart; ${ }^{\mathrm{D}}$ significantly different from frontal cortex; SEM, standard error of the mean. Greyscale bars represent frontal cortex data from our previous report (adapted from Pandya et al. [23], with permission).

heart, there was an apparent decrease in State $V_{2}$ respiration from $12 \mathrm{M}$ to $24 \mathrm{M}$ that did not reach significance. There was a significant increase in State $V_{2}$ from $1 \mathrm{M}$ to $12 \mathrm{M}$ in the liver. In the lung, just as in all other mitochondrial bioenergetic parameters, there was a significant decrease in State $V_{2}$ respiration after $1 \mathrm{M}$ across all other ages. It is interesting to note that the State $\mathrm{V}_{2}$ activity remained identical in heart across age group.

3.2. Age- and Organ-Specific Differences in Mitochondrial Enzymes. PDHC is the key regulatory enzyme of cellular metabolism because it links the Krebs cycle and subsequent oxidative phosphorylation with glycolysis and gluconeogenesis. In addition to mitochondrial respiratory parameters, we also analyzed PDHC enzyme activity (Figure 3(a)) in three visceral organs across life stages and compared them with the frontal cortex. Two-way ANOVA analysis indicated a significant interaction of age and organ on PDHC enzyme activity $\left(F_{9,24}=3.881 ; p=0.0005\right)$. The differences driving this effect appeared to be the high PDHC activity in the heart and the much lower activities across both the liver and lung. In the heart, which had the highest levels of PDHC activity, there was significantly increased and sustained enzymatic activity after $1 \mathrm{M}$. In the liver, there was a trend of decreasing PDHC enzyme activity with significant differences between $1 \mathrm{M}$ to $24 \mathrm{M}$ and $4 \mathrm{M}$ to $24 \mathrm{M}$. In the lung, PDHC activity remained higher from $1 \mathrm{M}$ to $4 \mathrm{M}$ and then there was a significant drop at $12 \mathrm{M}$ and $24 \mathrm{M}$.

Mitochondrial Complex I (Figure 3(b)), which is the primary entry point to the ETC, varied across organ with a significant main effect of organ $\left(F_{3,64}=11.74, p=3.19 e^{-6}\right)$. The highest Complex I enzyme activities were found in the heart, similar to PDHC activity. Within all other organ groups, there were no significant effects of age; however, there was a trend consistent with other measures of mitochondrial bioenergetics. For instance, analogous to State $V_{1}$ and PDHC, the Complex I activities in the lung and liver were considerably less than in heart.

Mitochondrial Complex II (Figure 4(a)), which is a secondary entry point to the ETC as well as a component of the Krebs cycle, was variable across organs. Two-way ANOVA indicated a significant interaction of age and organ in Complex II activity $\left(F_{9,64}=8.257 ; p=4.915 e^{-8}\right)$. Post hoc multiple comparisons indicated that all Complex II enzyme activity in the lung was significantly less compared to nearly all other organs. Within each organ, the effect of age was nearly identical to that of State $V_{2}$ (Figure 2(b)) or Complex II driven maximal respiration. In the heart, there was a significant trend of decreasing Complex II activity after $1 \mathrm{M}$. In the liver, there was a trend of increasing Complex II activity from $1 \mathrm{M}$ to $12 \mathrm{M}$ where the difference reached significance and plateau throughout $24 \mathrm{M}$. This effect was also captured in the State $\mathrm{V}_{2}$ respiration measures within the liver. In the lung, Complex II activity was highest at $1 \mathrm{M}$ and then significantly decreased thereafter in $4 \mathrm{M}$ and $12 \mathrm{M}$.

Mitochondrial Complex IV (Figure 4(b)), the last enzyme in the ETC, was also variable across organs, and twoway ANOVA analysis found significant main effects of age $\left(F_{3,64}=4.95 ; p=0.0037\right)$ and organ $\left(F_{3,64}=95.1 ; p=1.523 e^{-23}\right)$. Post hoc multiple comparisons indicated that all Complex IV enzyme activity in the lung and liver was significantly less compared to the heart. In the heart, there was a bellshaped trend of Complex IV activity across age, where 4M showed higher activity than other age groups. In the liver, there was a significant transient increase in Complex IV from $1 \mathrm{M}$ to $4 \mathrm{M}$, with a return to $1 \mathrm{M}$ activity levels from 


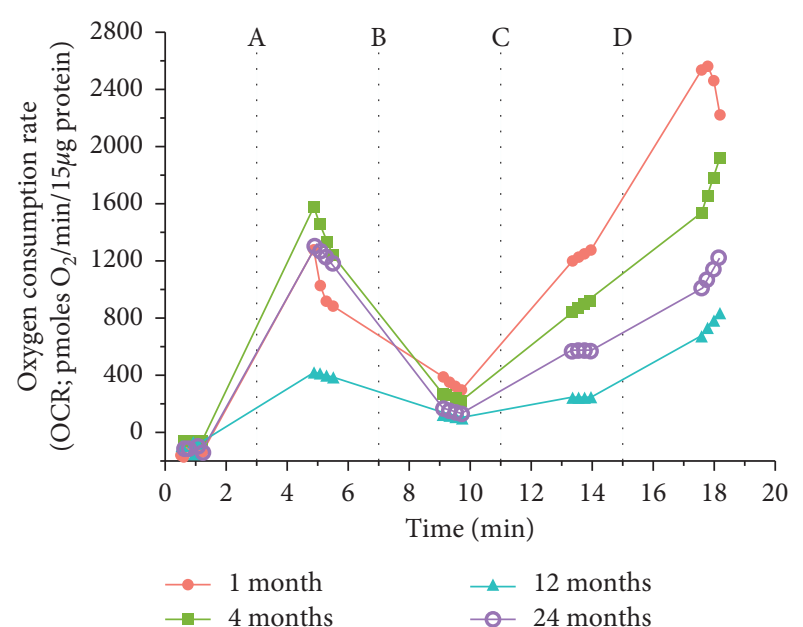

FIgURE 5: Typical traces of brain (frontal cortex) mitochondrial bioenergetics using Seahorse Extracellular Flux Analyzer from rats that are 1 month, 4 months, 12 months, and 24 months of age. Each line shown is representative point-to-point display of patterns of OCR respiration rates at $1,4,12$, or 24 months of age. Oxygen consumption rates (OCR) were measured with addition of substrates/inhibitors of electron transport chain. Vertical lines represent addition of compounds used to determine the various respiration states: (A) substrates (pyruvate, malate, and adenosine diphosphate) for State III; (B) oligomycin for State IV; (C) carbonyl cyanide 4-(trifluoromethoxy)phenylhydrazone (FCCP) for State $\mathrm{V}_{1}$; and (D) succinate and rotenone for State $\mathrm{V}_{2}$.

$12 \mathrm{M}$ to $24 \mathrm{M}$. In the lung, there was a significant and sustained increase in Complex IV activity after $1 \mathrm{M}$.

\section{Discussion}

Aging is a multifactorial process thought to be influenced by internal factors (e.g., genetics, stress, and nutrition) and external factors (e.g., lifestyle and environmental toxicants). There are several theories regarding the mechanism of aging which, based on their core assumptions, can be grouped into two categories: (1) aging is programmed or (2) aging is caused by an accumulation of damage [41]. Over the decades, there has been an increase in the understanding of cause vs. effect relationship in aging. In its inception, the free radical theory of aging is specifically limited to oxidative damage imparted by free radicals. The next evolution of that idea, the mitochondrial theory of aging, expanded the scope of damage to include any source of mitochondrial dysfunction such as increased mitochondrial electron leakage, mtDNA mutations, oxidative stress, or breakdown in the membrane potential to underline the cause of aging. More recently, it has been postulated [9] that mitochondrial bioenergetic dysfunction may be only one of many types of damage that accumulate from the imperfectness of biological systems over time. Other factors such as protein turnover rates may also contribute to the overall cellular bioenergetics capacity, thereby influencing the aging process [42]. Whichever doctrine one adheres to, it is undeniable that age-dependent decline in mitochondrial dysfunction does occur and does contribute to senescence. Whether or not mitochondrial dysfunction is the driving force of aging, the fact that environmental toxicants commonly interact with mitochondrial processes imposes an added threat to aging individuals. Current study was designed to extend our understanding based on current hypothesis that different organs may have altered metabolic energy demand and susceptibility/resilience to toxic chemicals within or across ages and therefore may contribute differently during altered life stages. To test whether energy metabolism differs in different organs and to investigate whether that metabolism changes over life span, we have examined mitochondrial function at various ages in a variety of organs associated with specific age-related deficits using a rat model of aging. Overall, current data indicated heart as the highly active metabolic organ followed by liver, and lung remained as the most vulnerable organ when considering the interactions of age-related sensitivities with exposure to chemical stressors from the environment perspective. Ultimately, these data may serve as a basic platform to test various stressors or toxicant effects on age- and organ-specific loss of homeostatic function.

In our previous study [23], we sought to characterize mitochondrial bioenergetic changes over time in various brain regions using an identical experimental condition in the identical age groups using Brown Norway rats. We found brain region-specific changes in the brain mitochondrial bioenergetics function over life stages. Given the susceptibility of mitochondria to environmental toxicants, we found these outcomes provided essential contextual information in the understanding of brain region susceptibility to environmental chemicals/toxicants during aging. This current study was designed to expand our understanding by characterizing visceral organ-specific mitochondrial bioenergetics at various life stages. We picked three visceral organs (i.e., heart, liver, and lung) which are considered to have higher metabolic capacities and critical targets to environmental chemicals following acute or chronic exposures. Based on current experimental data, we may better understand the metabolic capacity of these organs over age. This study may serve as a platform to screen various chemicals/toxic compounds based on their effects on mitochondrial respiration and metabolism over time. Additionally, these mitochondrial targets may be useful to delay progression of metabolic dysregulation across various life stages.

Mitochondrial bioenergetics and complex enzyme activities were studied in different organs and age groups using identical procedures. As described above, we found that for many mitochondrial parameters, age and organ interact to produce varying patterns of respiration and enzyme activities. The general pattern in the mitochondrial respiratory parameters (State III, IV, $V_{1}$, and $V_{2}$ respiration rates) across organ and over lifespan suggest that respiration is typically greatest in younger animals (1M) and then decreases over time until old age (24M). This pattern was not evident in all cases such as in the liver, where young animals (1M) had the lowest respiration rates of all parameters (State III, IV, $V_{1}$, and $V_{2}$ ) and middle-aged (12M) or old animals (24M) had the highest levels of respiration. 
This may be related to the development of that particular organ.

An interesting deviation from the general pattern of respiration can be seen in rates of State III and State $V_{1}$ in the heart where the usual decline in respiration only lasted until middle-age $(12 \mathrm{M})$ but was then followed by an apparent rebound effect in old animals (24M). A similar pattern was seen previously in frontal cortex in both State IV and State $\mathrm{V}_{1}$ respiration rates [23]. Together both increasing State III (related to ATP synthesis) and State $\mathrm{V}_{1}$ (Complex I driven maximum electron transport rates) represent increasing Complex I substrate driven ATP synthesis rates. In further support of there being an increase in Complex I driven State III respiration in the heart is the increase in PDHC activity throughout the life of the animal. An increase in PDHC activity indicates more Complex I substrates could be available to compensate decreased ATP synthesis rates between $1 \mathrm{M}$ to $12 \mathrm{M}$ period. As previously shown, PDHC activity in the frontal cortex remained stable throughout the lifetime of the animal. Consistent with the mitochondrial theory of aging, it is not surprising to see a decline of ATP synthesis capacity with age in the heart which could indicate less efficient mitochondria over time. However, the rebound in State III rates is quite surprising which suggests a possible compensatory mechanism operated at old age specifically in the heart and frontal cortex. To our knowledge, no such compensatory mechanism as described above has been reported in the literature. This compensation could be a way for the body to prioritize specific organs energy requirements during the onset of senescence. We find this prioritization scheme likely as we show a similar trend in respiration rates (frontal cortex $\sim$ heart $>$ liver $>$ lung) in each vital organ across age groups.

In the liver, we found a decreasing respiratory control ratio over lifetime. The higher State III rates in the liver indicated increased metabolic demand starting in middleage $(12 \mathrm{M})$. Meanwhile, some of the highest levels of proton leakage were detected in the liver at the same age (12M) and suggest in stark increase in uncoupling compared to younger animals. Furthermore, liver samples starting at $12 \mathrm{M}$ had the highest rates of Complex II driven uncoupling and significantly higher Complex II enzyme activity. This profile could be interpreted as reactive instead of proactive. Similar to this line, Serviddio et al. have previously reported increased uncoupling and proton leak in liver mitochondrial samples with aging, together with increased Complex I driven State III and State IV rates in liver in aged samples as compared to young group [43]. Moreover, cellular senescence drives agedependent hepatic steatosis (fatty liver) [44], and the increase in free fatty acids has been shown to increase expression of certain uncoupling proteins in mitochondria [45]. Therefore, it is possible that the increase in leakage that is driving the age-related decrease in respiratory control may be related to the onset of fatty liver disease as described by others. In the present study, we did not assess fatty liver status. Future studies should assess liver mitochondrial uncoupling more in depth by activation with free fatty acids.

Activation of PDHC promotes glucose utilization for energy production, whereas inactivation conserves glucose.
In the aging heart, it has been demonstrated that increased glucose utilization, via transgenic overexpression of glucose transporters in cardiomyocytes, produces a favorable metabolic phenotype that provides protections against ischemia [46]. In the heart (Figure 3(a)), there is a profound increase in glucose utilization as the animal ages. Perhaps, as suggested by Luptak et al., there exists a mechanism in the heart whereby increased glucose consumption can tender some protection against age-related complications. However, there is no further evidence of protection; in fact, there is quite the opposite. As glucose metabolism increases in the heart both ATP synthesis (State III) and Complex I driven maximal electron transport (State $V_{1}$ ) decrease with age suggesting a breakdown in the transfer of electrons though the ETC. The higher PDHC activity may be a compensatory mechanism as we saw decreased ATP synthesis (state III respiration) with aging. Heart PDHC may have to overwork to compensate for age related energy loss. This aging-related breakdown of the ETC is further supported by the drastic increase in proton leakage (State IV) in the heart though life span. Whether this phenotype is suggestive of age-related heart dysfunction is not known.

\section{Conclusions}

In summary, we report differential age- and organ-specific changes in the Brown Norway rat mitochondrial function. In combination with our previous report [23] the brain region-, age-, and organ-specific patterns reported in our studies may serve as a guideline for assessing the relative vulnerability of specific organs to environmental chemical insult across life span.

\section{Data Availability}

The data used to support the findings of the study are available from the corresponding author upon request.

\section{Disclosure}

The research described in this article has been reviewed by the National Health and Environmental Effects Research Laboratory, U.S. Environmental Protection Agency, and approved for publication. Approval does not signify that the contents necessarily reflect the views and policies of the Agency, nor does the mention of trade names of commercial products constitute endorsement or recommendation for use.

\section{Conflicts of Interest}

The authors declare that they have no conflicts of interest.

\section{Authors' Contributions}

Jignesh D. Pandya and Matthew Valdez contributed equally to this work. Jignesh D. Pandya carried out tissue isolations and seahorse experiments, calculated data, and wrote the manuscript. Matthew Valdez performed statistical analysis and wrote the manuscript. Joyce E. Royland proofread the 
manuscript and designed the experiments. Robert C. MacPhail designed the experiments and reviewed the manuscript. Patrick G. Sullivan designed experiments and reviewed manuscript. Prasada Rao S. Kodavanti conducted experiments, supervised the study, and proofread the manuscript. All authors read and approved the manuscript.

\section{Acknowledgments}

This study was supported by US-EPA Contract RFQ-RT-12140 (JDP and PGS, Co-PIs) and NIH grants NS062993 (PGS) and NS069633 (PGS). The authors would like to thank Andrea Sebastian for expert technical assistance. Drs. Michael F. Hughes and Colette Miller from the Integrated Systems Toxicity Division (ISTD) and Environmental Public Health Division (EPHD), respectively, of the National Health and Environmental Effects Research Laboratory (NHEERL) at the U.S. Environmental Protection Agency are acknowledged for their helpful comments on an earlier version of this manuscript.

\section{References}

[1] E. D. Hall, "The contributing role of lipid peroxidation and protein oxidation in the course of CNS injury neurodegeneration and neuroprotection: an overview," in Brain Neurotrauma Mol. Neuropsychol. Rehabil. Asp., F. H. Kobeissy, Ed., CRC Press/Taylor \& Francis, Boca Raton, FL, USA, 2015.

[2] M. P. Murphy, "How mitochondria produce reactive oxygen species," Biochemical Journal, vol. 417, no. 1, pp. 1-13, 2009.

[3] M. Schieber and N. S. Chandel, "ROS function in redox signaling and oxidative stress," Current Biology, vol. 24, pp. R453-R462, 2014.

[4] F. A. Lints, "The rate of living theory revisited," Gerontology, vol. 35, no. 1, pp. 36-57, 1989.

[5] D. Harman, "The biologic clock: the mitochondria?" Journal of the American Geriatrics Society, vol. 20, no. 4, pp. 145-147, 1972.

[6] M. D. Brand, "Uncoupling to survive? The role of mitochondrial inefficiency in ageing," Experimental Gerontology, vol. 35, no. 6-7, pp. 811-820, 2000.

[7] P. C. Tapia, "Sublethal mitochondrial stress with an attendant stoichiometric augmentation of reactive oxygen species may precipitate many of the beneficial alterations in cellular physiology produced by caloric restriction, intermittent fasting, exercise and dietary phytonutrients: "Mitohormesis" for health and vitality," Medical Hypotheses, vol. 66, no. 4, pp. 832-843, 2006.

[8] D. Harman, "Origin and evolution of the free radical theory of aging: a brief personal history, 1954-2009," Biogerontology, vol. 10, pp. 773-781, 2009.

[9] V. N. Gladyshev, "The free radical theory of aging is dead. Long live the damage theory!" Antioxidants \& Redox Signaling, vol. 20, no. 4, pp. 727-731, 2014.

[10] J. N. Meyer, J. H. Hartman, and D. F. Mello, "Mitochondrial toxicity," Toxicological Sciences, vol. 162, no. 1, pp. 15-23, 2018.

[11] K. B. Wallace, "Historical perspective of mitochondria in the toxicological sciences," Toxicological Sciences, vol. 162, no. 1, pp. 12-14, 2018.

[12] L. P. Wills, G. C. Beeson, D. B. Hoover, R. G. Schnellmann, and C. C. Beeson, "Assessment of ToxCast phase II for mitochondrial liabilities using a high-throughput respirometric assay," Toxicological Sciences, vol. 146, no. 2, pp. 226-234, 2015.

[13] P. Buffa, G. F. Azzone, E. Carafoli, and U. Muscatelo, "The mitochondrial biochemical lesion in pentachlorophenol intoxication," Bollettino della Societa Italiano di Biologia Sperimentale, vol. 35, pp. 1816-1820, 1959.

[14] T. Villa, L. Andri, and F. Brasca, "Enzymatic research at the mitochondrial level in experimental carbon monoxide poisoning Behavior of the cytochrome oxidases, aldolases and glutamic-oxalacetic and glutamic-pyruvic transaminases in the hepatic and renal mitochondria," Folia Medica Folia Medica Naples Italy, vol. 44, pp. 486-495, 1961.

[15] J. T. Greenamyre, R. Betarbet, and T. B. Sherer, "The rotenone model of Parkinson's disease: genes, environment and mitochondria," Parkinsonism \& Related Disorders, vol. 9, pp. 59-64, 2003.

[16] J. B. Chappell and G. D. Greville, "Effects of oligomycin on respiration and swelling of isolated liver mitochondria," Nature, vol. 190, no. 4775, pp. 502-504, 1961.

[17] A. P. West, "Mitochondrial dysfunction as a trigger of innate immune responses and inflammation," Toxicology, vol. 391, pp. 54-63, 2017.

[18] D. M. Gash, K. Rutland, N. L. Hudson et al., "Trichloroethylene: Parkinsonism and complex 1 mitochondrial neurotoxicity," Annals of Neurology, vol. 63, no. 2, pp. 184-192, 2008.

[19] A. Sauerbeck, R. Hunter, G. Bing, and P. G. Sullivan, "Traumatic brain injury and trichloroethylene exposure interact and produce functional, histological, and mitochondrial deficits," Experimental Neurology, vol. 234, no. 1, pp. 85-94, 2012.

[20] B. Zhou and R. Tian, "Mitochondrial dysfunction in pathophysiology of heart failure," Journal of Clinical Investigation, vol. 128, no. 9, pp. 3716-3726, 2018.

[21] S. M. Cloonan and A. M. K. Choi, "Mitochondria in lung disease," Journal of Clinical Investigation, vol. 126, no. 3, pp. 809-820, 2016.

[22] Y. Wei, R. S. Rector, J. P. Thyfault, and J. A. Ibdah, "Nonalcoholic fatty liver disease and mitochondrial dysfunction," World Journal of Gastroenterology, vol. 14, no. 2, p. 193, 2008.

[23] J. D. Pandya, J. E. Royland, R. C. MacPhail, P. G. Sullivan, and P. R. S. Kodavanti, "Age- and brain region-specific differences in mitochondrial bioenergetics in Brown Norway rats," Neurobiology of Aging, vol. 42, pp. 25-34, 2016.

[24] J. D. Pandya, J. R. Pauly, V. N. Nukala et al., "Post-injury administration of mitochondrial uncouplers increases tissue sparing and improves behavioral outcome following traumatic brain injury in rodents," Journal of Neurotrauma, vol. 24, no. 5, pp. 798-811, 2007.

[25] J. D. Pandya, J. R. Pauly, and P. G. Sullivan, "The optimal dosage and window of opportunity to maintain mitochondrial homeostasis following traumatic brain injury using the uncoupler FCCP," Experimental Neurology, vol. 218, no. 2, pp. 381-389, 2009.

[26] J. D. Pandya, P. G. Sullivan, and L. C. Pettigrew, "Focal cerebral ischemia and mitochondrial dysfunction in the TNFatransgenic rat," Brain Research, vol. 1384, pp. 151-160, 2011.

[27] P. G. Sullivan, S. Krishnamurthy, S. P. Patel, J. D. Pandya, and A. G. Rabchevsky, "Temporal characterization of mitochondrial bioenergetics after spinal cord injury," Journal of Neurotrauma, vol. 24, no. 6, pp. 991-999, 2007.

[28] J. D. Pandya, R. D. Readnower, S. P. Patel et al., "N-acetylcysteine amide confers neuroprotection, improves bioenergetics and behavioral outcome following TBI," Experimental Neurology, vol. 257, pp. 106-113, 2014. 
[29] J. D. Pandya, P. G. Sullivan, L. Y. Leung, F. C. Tortella, D. A. Shear, and Y. Deng-Bryant, "Advanced and high-throughput method for mitochondrial bioenergetics evaluation in neurotrauma," Methods in Molecular Biology, vol. 1462, pp. 597-610, 2016.

[30] S. P. Patel, P. G. Sullivan, J. D. Pandya et al., "N-acetylcysteine amide preserves mitochondrial bioenergetics and improves functional recovery following spinal trauma," Experimental Neurology, vol. 257, pp. 95-105, 2014.

[31] A. Sauerbeck, J. Pandya, I. Singh et al., "Analysis of regional brain mitochondrial bioenergetics and susceptibility to mitochondrial inhibition utilizing a microplate based system," Journal of Neuroscience Methods, vol. 198, no. 1, pp. 36-43, 2011.

[32] J. D. Pandya, V. N. Nukala, and P. G. Sullivan, "Concentration dependent effect of calcium on brain mitochondrial bioenergetics and oxidative stress parameters," Front. Neuroenergetics, vol. 5, 2013.

[33] J. D. Pandya, R. Grondin, H. M. Yonutas et al., "Decreased mitochondrial bioenergetics and calcium buffering capacity in the basal ganglia correlates with motor deficits in a nonhuman primate model of aging," Neurobiology of Aging, vol. 36, no. 5, pp. 1903-1913, 2015.

[34] J. D. Pandya, L. Y. Leung, X. Yang et al., "Comprehensive profile of acute mitochondrial dysfunction in a preclinical model of severe penetrating TBI," Frontiers in Neurology, vol. 10, p. 605, 2019.

[35] D. Ziegler and J. S. Rieske, "Preparation and properties of succinate dehydrogenase-coenzyme Q reductase (complex II)," Methods in Enzymology, vol. 10, pp. 231-235, 1967.

[36] D. C. Wharton and A. Tzagoloff, "Cytochrome oxidase from beef heart mitochondria," Methods in Enzymology, vol. 10, pp. 245-250, 1967.

[37] Team RStudio, RStudio, Integrated Development for R, RStudio, Inc., Boston, MA, USA, 2015.

[38] H. Wickham, R. François, L. Henry, and K. Müller, RStudio, dplyr: A Grammar of Data Manipulation, Integrated Development for R, RStudio, Inc., Boston, MA, USA, 2018.

[39] H. Wickham, W. Chang, L. Henry et al., RStudio, Ggplot2: Create Elegant Data Visualisations Using the Grammar of Graphics, Integrated Development for R, RStudio, Inc., Boston, MA, USA, 2018.

[40] C. O. Wilke, "Cowplot: streamlined plot theme and plot annotations for "Ggplot2"," 2018.

[41] P. V. Sergiev, O. A. Dontsova, and G. V. Berezkin, "Theories of aging: an ever-evolving field," Acta Naturae, vol. 7, no. 1, pp. 9-18, 2015.

[42] A. Popa-Wagner, R. E. Sandu, C. Cristin et al., "Increased degradation rates in the components of the mitochondrial oxidative phosphorylation chain in the cerebellum of old mice," Frontiers in Aging Neuroscience, vol. 10, p. 32, 2018.

[43] G. Serviddio, F. Bellanti, A. D. Romano et al., "Bioenergetics in aging: mitochondrial proton leak in aging rat liver, kidney and heart," Redox Report, vol. 12, no. 1-2, pp. 91-95, 2007.

[44] M. Ogrodnik, S. Miwa, T. Tchkonia et al., "Cellular senescence drives age-dependent hepatic steatosis," Nature Communications, vol. 8, 2017.

[45] X. Jin, Z. Xiang, Y. Chen, K. Ma, Y. Ye, and Y. Li, "Uncoupling protein and nonalcoholic fatty liver disease," Chinese Medical Journal, vol. 126, no. 16, pp. 3151-3155, 2013.

[46] I. Luptak, J. Yan, L. Cui, M. Jain, R. Liao, and R. Tian, "Longterm effects of increased glucose entry on mouse hearts during normal aging and ischemic stress," Circulation, vol. 116, no. 8, pp. 901-909, 2007. 